TRANSACTIONS OF THE

AMERICAN MATHEMATICAL SOCIETY

Volume 357, Number 1, Pages 385-396

S 0002-9947(04)03510-X

Article electronically published on August 11, 2004

\title{
LACK OF NATURAL WEIGHTED ESTIMATES FOR SOME SINGULAR INTEGRAL OPERATORS
}

\author{
JOSÉ MARÍA MARTELL, CARLOS PÉREZ, AND RODRIGO TRUJILLO-GONZÁLEZ
}

AbSTRACT. We show that the classical Hörmander condition, or analogously the $L^{r}$-Hörmander condition, for singular integral operators $T$ is not sufficient to derive Coifman's inequality

$$
\int_{\mathbb{R}^{n}}|T f(x)|^{p} w(x) d x \leq C \int_{\mathbb{R}^{n}} M f(x)^{p} w(x) d x,
$$

where $0<p<\infty, M$ is the Hardy-Littlewood maximal operator, $w$ is any $A_{\infty}$ weight and $C$ is a constant depending upon $p$ and the $A_{\infty}$ constant of $w$. This estimate is well known to hold when $T$ is a Calderón-Zygmund operator.

As a consequence we deduce that the following estimate does not hold:

$$
\int_{\mathbb{R}^{n}}|T f(x)|^{p} w(x) d x \leq C \int_{\mathbb{R}^{n}} M f(x)^{p} M w(x) d x,
$$

where $0<p \leq 1$ and where $w$ is an arbitrary weight. However, by a recent result due to $\bar{A}$. Lerner, this inequality is satisfied whenever $T$ is a CalderónZygmund operator.

One of the main ingredients of the proof is a very general extrapolation theorem for $A_{\infty}$ weights.

\section{INTRODUCTION}

There is a principle in the classical Calderón-Zygmund theory which roughly states that any singular integral operator is controlled by a suitable maximal operator. The model example is provided by the Calderón-Zygmund singular integral operators which, as it is well known, are controlled by the Hardy-Littlewood maximal function $M$. An explicit way of expressing this principle is by means of the following estimate due to Coifman [Coi] (see also [Ste, p. 205]): let $T$ be any Calderón-Zygmund operator and let $0<p<\infty$. Then there exists a constant $C$ such that for any $w \in A_{\infty}$,

$$
\int_{\mathbb{R}^{n}}|T f(x)|^{p} w(x) d x \leq C \int_{\mathbb{R}^{n}} M f(x)^{p} w(x) d x,
$$

for every $f$ such that the left-hand side is finite. We refer to [Duo] or [Chr] for the precise definitions.

Received by the editors May 23, 2003 and, in revised form, September 18, 2003.

2000 Mathematics Subject Classification. Primary 42B20, 42B25.

Key words and phrases. Calderón-Zygmund singular integral operators, Muckenhoupt weights, maximal functions.

The first author was partially supported by MCYT Grant BFM2001-0189.

The second author was partially supported by DGICYT Grant PB980106.

The third author was supported by MCYT Grant BFM2002-02098.

(C)2004 American Mathematical Society 
These types of inequalities encode a good amount of information about the behavior of such operators. For instance, when $p>1$ and $w \in A_{p}$, we can apply Muckenhoupt's theorem (see [Duo]) to obtain that $T$ is bounded on $L^{p}(w)$. Also, estimate (C) combined with some sharp two weight norm inequalities for the Hardy-Littlewood maximal function from [Pe2], yields the following sharp weighted inequality for $T$ and $p>1$ :

$$
\int_{\mathbb{R}^{n}}|T f(x)|^{p} w(x) d x \leq C \int_{\mathbb{R}^{n}}|f(x)|^{p} M^{[p]+1} w(x) d x,
$$

where no assumption is assumed on $w$. This was shown in Pe1, generalizing some partial result (by a different method) due to $\mathrm{M}$. Wilson in Wil. As a consequence one can show that for any $\epsilon>0$ (see [Pe1]),

$$
w\left(\left\{x \in \mathbb{R}^{n}:|T f(x)|>\lambda\right\}\right) \leq \frac{C_{\epsilon}}{\lambda} \int_{\mathbb{R}^{n}}|f| M_{L(\log L)^{\epsilon}}(w) d x,
$$

where, again, no assumption is assumed on $w$.

As is well known, estimate (C) is proved by means of a good- $\lambda$ inequality (or relative distributional inequality) between $T$ and $M$. However, by very recent results it is possible to avoid this classical technique. Indeed, there is an interesting estimate due to A. Lerner [Ler] which establishes

$$
\int_{\mathbb{R}^{n}} T f(x) w(x) d x \leq C \int_{\mathbb{R}^{n}} M f(x) M w(x) d x,
$$

for any Calderón-Zygmund operator $T$ and any arbitrary weight $w$. Here it is only assumed that $w$ is an a.e. non-negative locally integrable function. Lerner's inequality is proved by means of the so-called local sharp maximal operator and without the good- $\lambda$ method. The local sharp maximal operator is an interesting operator introduced in [S] by J.-O. Strömberg motivated by the work of F. John in [J]. Pushing the techniques in [Ler one can get the same estimate (1) with exponents $0<p \leq 1$. Taking, in particular, $w \in A_{1}$, which means $M w(x) \leq C w(x)$ for a.e. $x \in \mathbb{R}^{n}$, we obtain

$$
\int_{\mathbb{R}^{n}}|T f(x)|^{p} w(x) d x \leq C \int_{\mathbb{R}^{n}} M f(x)^{p} w(x) d x,
$$

for any $0<p \leq 1$ and for all $w \in A_{1}$. This estimate plus an extrapolation result in $\mathrm{CMP}$ (see Theorem 4.1 estimate $(c)$ below) provide $(\mathrm{C})$. We would like to emphasize that this way, which combines the ideas of [Ler] and CMP], yields a new proof of Coifman's estimate without use of the good- $\lambda$ technique.

\section{KNOWN RESULTS}

We recall the definitions of the $A_{p}$ classes of weights of Muckenhoupt. For more details the reader is referred to [Duo] or $\mathrm{GR}$ ]. A weight $w$ belongs to the class $A_{p}$, $1<p<\infty$, if there is a constant $C$ such that

$$
\left(\frac{1}{|Q|} \int_{Q} w(y) d y\right)\left(\frac{1}{|Q|} \int_{Q} w(y)^{1-p^{\prime}} d y\right)^{p-1} \leq C
$$

for each cube $Q$ (with sides parallel to the coordinate axes in the sequel). A weight $w$ belongs to the class $A_{1}$ if there is a constant $C$ such that

$$
\frac{1}{|Q|} \int_{Q} w(y) d y \leq C w(x), \quad \text { for a.e. } x \in Q \text {. }
$$


Equivalently $w \in A_{1}$ if and only if

$$
M w(x) \leq C w(x), \quad \text { for a.e. } x \in \mathbb{R}^{n} .
$$

These classes are increasing and therefore it is natural to define the $A_{\infty}$ class of weights as follows:

$$
A_{\infty}=\bigcup_{p>1} A_{p}
$$

which, by Muckenhoupt's characterization, turns out to be equivalent to

$$
\frac{w(E)}{w(Q)} \leq c\left(\frac{|E|}{|Q|}\right)^{\rho}
$$

for some $c$ and $\rho$ and independent of $Q$ and $E \subset Q$.

Throughout this paper $T$ will denote a singular integral operator of convolution type, that is,

$$
T f(x)=p \cdot v \cdot \int_{\mathbb{R}^{n}} K(x-y) f(y) d y
$$

with kernel $K$ having bounded Fourier transform $\widehat{K} \in L^{\infty}\left(\mathbb{R}^{n}\right)$ so that $T$ is bounded on $L^{2}\left(\mathbb{R}^{n}\right)$.

The key condition in the proof of $(\mathrm{C})$ is that the kernel satisfies the classical Lipschitz condition: there are positive numbers $\alpha, c$ such that whenever $|x|>c|y|$,

$$
|K(x-y)-K(x)| \leq C \frac{|y|^{\alpha}}{|x|^{\alpha+n}} .
$$

The purpose of this paper is to show that, however, there is no analogue of $(\mathrm{C})$ when we relax (21) by the more general Hörmander condition:

$$
\sup _{y \in \mathbb{R}^{n}} \int_{|x|>c|y|}|K(x-y)-K(x)| d x<\infty .
$$

We even prove that in (C), $M$ cannot be replaced by the pointwise bigger maximal operator $M_{t}, t \geq 1$, where $M_{t} f(x)=\left(M\left(|f|^{t}\right)(x)\right)^{1 / t}$. In fact, we will see that (C) does not hold for certain intermediate conditions between (2) and (3). To be precise we consider a variant of the Hörmander condition (3).

Definition 2.1. Let $1 \leq r \leq \infty$. We say that the kernel $K$ verifies the $L^{r}$ Hörmander condition if there are positive numbers $c$ and $C_{r}$ such that for any $y \in \mathbb{R}^{n}$ and $R>c|y|$,

$$
\sum_{m=1}^{\infty}\left(2^{m} R\right)^{\frac{n}{r^{\prime}}}\left(\int_{2^{m}} R<|x| \leq 2^{m+1} R\left(K(x-y)-\left.K(x)\right|^{r} d x\right)^{\frac{1}{r}} \leq C_{r}\right.
$$

in the case $r<\infty$, and

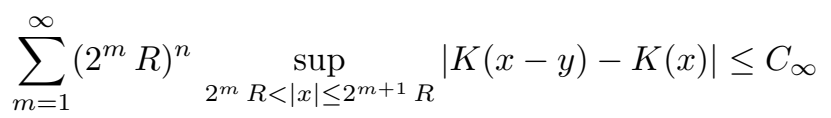

when $r=\infty$. We will write $H_{r}$ for the class of kernels satisfying the $L^{r}$-Hörmander condition.

This definition is implicit in the work of D. Kurtz and R. Wheeden [KW], where it is shown that the classical Dini condition for $K$ implies that $K \in H_{r}$ (cf. $\mathrm{KW}$ p. 359]). Later on, these classes $H_{r}$ were considered in [RRT and Wat. In fact, in this last paper the $L^{r}$-Hörmander condition plays an essential role when 
studying rough singular integral operators. Namely, for such an operator $T$, one can write $T=\sum T_{j}$, where the kernel of $T_{j}$ satisfies the $L^{r}$-Hörmander condition with constant growing linearly in $j$.

Observe that in the case $r=1, H_{1}$ is just the class of kernels verifying the classical Hörmander condition (3). It is clear that the $L^{r}$-Hörmander condition becomes stronger as $r$ increases, namely,

$$
H_{\infty}^{*} \subsetneq H_{\infty} \subsetneq H_{r} \subsetneq H_{1},
$$

where we denote by $H_{\infty}^{*}$ the class of kernels satisfying the classical Lipschitz condition (2). Thus, the operator $T$ with kernel $K \in H_{r}, r \geq 1$, is of weak type $(1,1)$ and bounded on $L^{p}\left(\mathbb{R}^{n}\right), 1<p<\infty$ (see for example [Duo] or [GR]).

For these classes of operators some weighted estimates in the spirit of $(\mathrm{C})$ are known.

Theorem 2.2. Let $T$ be a singular integral operator with kernel satisfying the $L^{r}-$ Hörmander condition. Then:

(i) If $1<r<\infty$ we have

$$
\int_{\mathbb{R}^{n}}|T f(x)|^{p} w(x) d x \leq C \int_{\mathbb{R}^{n}}\left(M_{r^{\prime}} f(x)\right)^{p} w(x) d x
$$

for $0<p<\infty$ and $w \in A_{\infty}$, whenever the left-hand side is finite. Furthermore, $T$ is bounded on $L^{p}(w)$ if $w \in A_{p / r^{\prime}}$ for $r^{\prime} \leq p<\infty$, or if $w^{1-p^{\prime}} \in A_{p^{\prime} / r^{\prime}}$ for $1<p \leq r$, or if $w^{r^{\prime}} \in A_{p}$ for $1<p<\infty$.

(ii) If $r=\infty$ we have

$$
\int_{\mathbb{R}^{n}}|T f(x)|^{p} w(x) d x \leq C \int_{\mathbb{R}^{n}}(M f(x))^{p} w(x) d x
$$

for $0<p<\infty$ and $w \in A_{\infty}$, whenever the left-hand side is finite. As a consequence, $T$ is bounded on $L^{p}(w)$, if $w \in A_{p}$ for $1<p<\infty$.

The proof of $(i)$ is based on the pointwise estimate

$$
M^{\sharp}(T f)(x) \leq c_{r} M_{r^{\prime}} f(x)
$$

and can be found in [RRT] (see also Wat]). Recall that the Fefferman-Stein sharp maximal function is defined as

$$
M^{\sharp} f(x)=\sup _{Q \ni x} \frac{1}{|Q|} \int_{Q}\left|f(y)-f_{Q}\right| d y,
$$

where $f_{Q}$ stands for the average of $f$ over $Q$. As a consequence, $T$ is bounded on $L^{p}(w)$ if $w \in A_{p / r^{\prime}}$ for $r^{\prime}<p<\infty$, or if $w^{1-p^{\prime}} \in A_{p^{\prime} / r^{\prime}}$ for $1<p<r$, or if $w^{r^{\prime}} \in A_{p}$ for $1<p<\infty$. The case $p=r^{\prime}$ is not so immediate, but it can be achieved by interpolation with change of measure and by the reverse Hölder property (see RRT for more details).

The proof of $(i i)$ that corresponds to $r=\infty$ arises from the pointwise estimate

$$
M_{\delta}^{\sharp}(T f)(x) \leq c_{\delta} M f(x)
$$

for any $0<\delta<1$, where $M_{\delta}^{\sharp} g(x)=\left(M^{\sharp}\left(|g|^{\delta}\right)(x)\right)^{1 / \delta}$. To get this inequality one should follow the same ideas. For details of this technique we refer the reader to AP and [PT]. Note that in this way we have recovered (C) for a bigger class of operators since, as we already mentioned, $H_{\infty}^{*} \subsetneq H_{\infty}$. An explicit example can be easily adapted from Lemma 6.1 by taking $K=\chi_{B_{1}(0)} \in H_{\infty}$ but not in $H_{\infty}^{*}$. 
The previous results drive us to the following questions:

- Is it possible to get similar estimates for $r=1$ ? That is, what is the substitute, if any, of $M_{r^{\prime}}$ in (4) when $K \in H_{1}$ ?

- For $1<r<\infty$, can we replace $M_{r^{\prime}}$ in (4) by the pointwise smaller operator $M_{t}$ with $1 \leq t<r^{\prime}$ ?

- Is the operator $T$ bounded on $L^{p}(w)$ for every $1<p<\infty$ and for every $w \in A_{p}$ or, even more, for $w \in A_{1}$ ?

In this paper we show that the answer to each of the above questions is negative.

\section{MAIN RESULTS}

The following theorem shows that (4) and the estimate $M^{\sharp}(T f)(x) \leq c_{r} M_{r^{\prime}} f(x)$ are sharp.

Theorem 3.1. Let $1 \leq r<\infty$ and $1 \leq t<r^{\prime}$. There exists a singular integral operator $T$ with kernel in $H_{r}$ for which the following estimate does not hold:

$$
\int_{\mathbb{R}^{n}}|T f(x)|^{p} w(x) d x \leq C \int_{\mathbb{R}^{n}}\left(M_{t} f(x)\right)^{p} w(x) d x,
$$

for any $f$ such that the left-hand side is finite where $0<p<\infty, w \in A_{\infty}$ and $C$ is a constant depending upon $p$ and the $A_{\infty}$ constant of $w$. Similarly, the inequality (6) does not hold when the strong $L^{p}(w)$ norms are replaced by the weak $L^{p, \infty}(w)$ norms in both sides, namely,

$$
\|T f\|_{L^{p, \infty}(w)} \leq C\left\|M_{t} f\right\|_{L^{p, \infty}(w)} .
$$

As a consequence we deduce that the following estimate also fails:

$$
\int_{\mathbb{R}^{n}}|T f(x)|^{p} w(x) d x \leq C \int_{\mathbb{R}^{n}}\left(M_{t} f(x)\right)^{p} M w(x) d x,
$$

where $0<p \leq 1$ and where $w$ is an arbitrary weight.

We pay special attention to the case $r=1$ corresponding to the classical Hörmander condition for which the theorem shows that there is no estimate similar to the model case $(\mathrm{C})$ as mentioned in the abstract.

Inequality (8) should be compared with the result due to Lerner which states that this inequality holds with $t=1$ whenever the kernel is in $H_{\infty}^{*}$; see [Ler] for more details.

The proof of Theorem 3.1 will follow from the following theorem for power weights and an appropriate extrapolation type result from [CMP] (see Theorem 4.1 below). The idea will be that if we disprove such an estimate for one fixed exponent $0<p_{0}<\infty$, then the same inequalities are false for the whole range of $p$ 's.

Theorem 3.2. Let $1 \leq r<\infty, 1 \leq p<r^{\prime}$ and $-n<\alpha<-n p / r^{\prime}$. There exists a singular integral operator $T$ with kernel in $H_{r}$ for which the following estimate does not hold:

$$
\|T f\|_{L^{p, \infty}\left(w_{\alpha}\right)} \leq C\|f\|_{L^{p}\left(w_{\alpha}\right)}
$$

where $w_{\alpha}(x)=|x|^{\alpha}$. 
This negative result should be compared with the following positive result: let $r, p$ be as in the theorem and let $-n p / r^{\prime}<\alpha \leq 0$. Then the following estimate holds:

$$
\|T f\|_{L^{p}(w)} \leq C\|f\|_{L^{p}(w)},
$$

where $w(x)=|x|^{\alpha}$. This arises essentially from the results by Watson Wat] using interpolation with change of measures.

Remark 3.3. We observe that in Theorem 3.1 the estimates do not hold even for the smaller class of weights $A_{1}$. This follows from the proof.

Corollary 3.4. Let $1 \leq r<\infty$. There exists a singular integral operator $T$ with kernel in $H_{r}$ for which the following estimate does not hold:

$$
\int_{\mathbb{R}^{n}}|T f(x)|^{p} w(x) d x \leq C \int_{\mathbb{R}^{n}}|f(x)|^{p} w(x) d x,
$$

where either $1<p<r^{\prime}, w \in A_{1}$, or $1<p<\infty, w \in A_{p}$.

Proof. The first statement is clear since the weights in Theorem 3.2 are in the class $A_{1}$. For the second we use the extrapolation theorem of Rubio de Francia (see [Duo p. 141]): if for some $1<p_{0}<\infty$, (11) holds for every $w \in A_{p_{0}}$, it is also true for any $1<p<\infty$ and for any $w \in A_{p}$, contradicting Theorem 3.2 as well.

We want to point out that in this result the range of exponents $1<p<r^{\prime}$ is sharp, since for $r^{\prime} \leq p<\infty$ and $w \in A_{1} \subset A_{p / r^{\prime}}$, the inequality (11) holds (see (4) above). Note that when $r=1$ no weighted estimate holds for $T$.

Remark 3.5. In the case of kernels satisfying the classical Hörmander condition (3), some counterexamples of weighted norm estimates for singular integral operators are given in [Hof]. In this paper, the author shows how (3) is not sufficient to derive strong and weak type estimates for power weights.

It should also be mentioned that in the work $[\mathrm{MW}]$ there are positive and negative results for weighted norm estimates for singular integral operators with rough kernels, namely with kernels satisfying no smoothness condition.

Remark 3.6. Condition (2) has also been used to prove the weak type $(1,1)$ for the supremum of the truncated integrals of Calderón-Zygmund operators. Although (3) is not sufficient for the weighted norm estimates, for the problem of the supremum of the truncated integrals it is, since in Gra it is shown that (3) guarantees that boundedness.

\section{Extrapolation Results}

One of the main ingredients in the proof of Theorem 3.1 is the strong use of some extrapolation type results which can be found in CMP. As a consequence we will see that to prove Theorem 3.1] it suffices to show that either (6) or the corresponding weak type-weak type do not hold for just one exponent $p_{0}$.

In what follows $G$ and $S$ are two operators defined on some class of smooth functions $\mathcal{S}$ such that $G f \geq 0, S f \geq 0$ for $f \in \mathcal{S}$. When we write an estimate like

$$
\|G f\|_{L^{p}(w)} \leq C\|S f\|_{L^{p}(w)},
$$

we understand that it holds for any $f \in \mathcal{S}$ such that the left-hand side is finite and that $C$ depends only upon the $A_{\infty}$ constant of $w$ and $p$. 
Theorem 4.1 ([CMP]). Let $G, S$ be as above. Consider the following estimates:

(a) $\|G f\|_{L^{p_{0}(w)}} \leq C\|S f\|_{L^{p_{0}(w)}}$, for some $0<p_{0}<\infty$ and all $w \in A_{\infty}$.

(b) $\|G f\|_{L^{p}(w)} \leq C\|S f\|_{L^{p}(w)}$, for all $0<p<\infty$ and all $w \in A_{\infty}$.

(c) $\|G f\|_{L^{p}(w)} \leq C\|S f\|_{L^{p}(w)}$, for all $0<p<p_{0}$, for some $p_{0}$, and all $w \in A_{1}$.

(d) $\|G f\|_{L^{p_{0}, \infty}(w)} \leq C\|S f\|_{L^{p_{0}, \infty}(w)}$, for some $0<p_{0}<\infty$ and all $w \in A_{\infty}$.

(e) $\|G f\|_{L^{p, \infty}(w)} \leq C\|S f\|_{L^{p, \infty}(w)}$, for all $0<p<\infty$ and all $w \in A_{\infty}$.

Then,

$$
(a) \Longleftrightarrow(b) \Longleftrightarrow(c) \Longrightarrow(e) \quad \text { and } \quad(d) \Longleftrightarrow(e)
$$

Here we will present a sketch of the proof of Theorem 4.1 for the sake of completeness. The reader is referred to the original source [CMP] for more details and for a great deal of applications.

Proof of Theorem 4.1. We are going to show that

$$
(a) \Longrightarrow(c) \Longrightarrow(b)
$$

and, since $(b)$ obviously implies $(a)$, we see that $(a),(b)$ and $(c)$ are equivalent.

$(a) \Longrightarrow(c)$ We will use the Rubio de Francia's algorithm (see [Duo]). We can assume that the left-hand side is positive and that the right-hand side is finite. Let $s=\frac{p_{0}}{p}>1$. Since $w \in A_{1} \subset A_{s^{\prime}}$ we have that $M$ is a continuous operator on $L^{s^{\prime}}(w)$. We denote the norm of $M$ as a bounded operator on this space by $\|M\|_{L^{s^{\prime}}(w)}$. For $0 \leq h \in L^{s^{\prime}}(w)$, we consider

$$
\mathcal{R} h=\sum_{k=0}^{\infty} \frac{M^{k} h}{2^{k}\|M\|_{L^{s^{\prime}}(w)}^{k}},
$$

where $M^{k}$ is the operator $M$ iterated $k$ times for $k \geq 1$ and for $k=0$ it is just the identity. From the definition of $\mathcal{R}$ it is immediate that:

(i) $h(x) \leq \mathcal{R} h(x)$.

(ii) $\|\mathcal{R} h\|_{L^{s^{\prime}}(w)} \leq 2\|h\|_{L^{s^{\prime}}(w)}$.

(iii) $M(\mathcal{R} h)(x) \leq 2\|M\|_{L^{s^{\prime}(w)}} \mathcal{R} h(x)$, and thus $\mathcal{R} h \in A_{1}$ with constant independent of $h$.

Now we take

$$
V f=\mathcal{R}\left(\left(\frac{G f}{\|G f\|_{L^{p}(w)}}\right)^{\frac{p}{s^{\prime}}}+\left(\frac{S f}{\|S f\|_{L^{p}(w)}}\right)^{\frac{p}{s^{\prime}}}\right) \in L^{s^{\prime}}(w) .
$$

Hölder's inequality leads us to obtain

$$
\begin{array}{rl}
\int_{\mathbb{R}^{n}} G & f(x)^{p} w(x) d x \\
\leq & \left(\int_{\mathbb{R}^{n}} G f(x)^{p_{0}} V f(x)^{-s} w(x) d x\right)^{\frac{1}{s}}\left(\int_{\mathbb{R}^{n}} V f(x)^{s^{\prime}} w(x) d x\right)^{\frac{1}{s^{\prime}}} \\
& =I \cdot I I .
\end{array}
$$

For the first term, note that the factorization theorem for weights allows us to get $w(V f)^{-s}=w(V f)^{1-(1+s)} \in A_{1+s} \subset A_{\infty}$ since $w, V f \in A_{1}$ (by $\left.(i i i)\right)$ and $1+s>1$. 
It is easy to check that we can apply $(a)$ since $I$ is finite. Note that $(i)$ implies

$$
\left(\frac{S f(x)}{\|S f\|_{L^{p}(w)}}\right)^{\frac{p}{s^{\prime}}} \leq V f(x) .
$$

Then, we can use this expression combined with $(a)$ to get

$$
I \leq C\left(\int_{\mathbb{R}^{n}} S f(x)^{p_{0}} V f(x)^{-s} w(x) d x\right)^{\frac{1}{s}} \leq C \int_{\mathbb{R}^{n}} S f(x)^{p} w(x) d x .
$$

For the second term we use (ii) to show that $I I \leq 4$.

$(c) \Longrightarrow(b)$ Let us fix $0<p<\infty$ and $w \in A_{\infty}$. Let us recall that the Muckenhoupt classes are increasing, that is, $A_{p_{1}} \subset A_{p_{2}}$ for $1 \leq p_{1} \leq p_{2}$. Then, there exists $0<q<\min \left\{p, p_{0}\right\}$ such that $w \in A_{r}$ for $r=\frac{p}{q}>1$. For $0 \leq h \in L^{r^{\prime}}\left(w^{1-r^{\prime}}\right)$, we use Rubio de Francia's algorithm in the following way:

$$
\mathcal{R} h(x)=\sum_{k=0}^{\infty} \frac{M^{k} h(x)}{2^{k}\|M\|_{L^{r^{\prime}}\left(w^{1-r^{\prime}}\right)}^{k}},
$$

where $\|M\|_{L^{r^{\prime}\left(w^{1-r^{\prime}}\right)}}$ denotes the norm of $M$ as a bounded operator on $L^{r^{\prime}}\left(w^{1-r^{\prime}}\right)$ which is finite since $w^{1-r^{\prime}} \in A_{r^{\prime}}$. Again, we have

(i) $h(x) \leq \mathcal{R} h(x)$.

(ii) $\|\mathcal{R} h\|_{L^{r^{\prime}}\left(w^{1-r^{\prime}}\right)} \leq 2\|h\|_{L^{r^{\prime}\left(w^{1-r^{\prime}}\right)}}$.

(iii) $M(\mathcal{R} h)(x) \leq 2\|M\|_{L^{r^{\prime}}\left(w^{1-r^{\prime}}\right)} \mathcal{R} h(x)$, and thus $\mathcal{R} h \in A_{1}$ with constant independent of $h$.

On the other hand,

$$
\|G f\|_{L^{p}(w)}^{q}=\left\|(G f)^{q}\right\|_{L^{r}(w)}=\sup _{h} \int_{\mathbb{R}^{n}} G f(x)^{q} h(x) w(x) d x,
$$

where the supremum is taken over all functions $0 \leq h \in L^{r^{\prime}}(w)$ with $\|h\|_{L^{r^{\prime}(w)}}=1$. Take such a function $h$. Then $h w \in L^{r^{\prime}}\left(w^{1-r^{\prime}}\right)$ and

$$
\|h w\|_{L^{r^{\prime}\left(w^{1-r^{\prime}}\right)}}=\|h\|_{L^{r^{\prime}(w)}}=1 .
$$

By using $(b)\left(0<q<p_{0}\right)$ with the weight $\mathcal{R}(h w) \in A_{1}$ and $(i)$, we obtain

$\int_{\mathbb{R}^{n}} G f(x)^{q} h(x) w(x) d x \leq \int_{\mathbb{R}^{n}} G f(x)^{q} \mathcal{R}(h w)(x) d x \leq C \int_{\mathbb{R}^{n}} S f(x)^{q} \mathcal{R}(h w)(x) d x$, provided the middle term is finite. By (ii) and Hölder's inequality we get

$$
\int_{\mathbb{R}^{n}} G f(x)^{q} \mathcal{R}(h w)(x) d x \leq\|G f\|_{L^{p}(w)}^{q}\|\mathcal{R}(h w)\|_{L^{r^{\prime}\left(w^{1-r^{\prime}}\right)}} \leq 2\|G f\|_{L^{p}(w)}^{q}<\infty .
$$

The previous computations also hold for $S f$ and it follows that

$$
\int_{\mathbb{R}^{n}} G f(x)^{q} h(x) w(x) d x \leq C \int_{\mathbb{R}^{n}} S f(x)^{q} \mathcal{R}(h w)(x) d x \leq C\|S f\|_{L^{p}(w)}^{q},
$$

which leads to the desired estimate.

$(c) \Longrightarrow(e)$ Let us fix $0<p<\infty$ and $w \in A_{\infty}$. There exists $0<q<p$ such that $w \in A_{r}$ for $r=\frac{p}{q}>1$. Then,

$$
\|G f\|_{L^{p, \infty}(w)}^{q}=\left\|(G f)^{q}\right\|_{L^{r, \infty}(w)}=\sup _{h} \int_{\mathbb{R}^{n}} G f(x)^{q} h(x) w(x) d x,
$$


where the supremum is taken over all functions $0 \leq h \in L^{r^{\prime}, 1}(w)$ such that $\|h\|_{L^{r^{\prime}, 1}(w)}=1$. We fix one of these functions $h$. Next, we consider the weighted Hardy-Littlewood maximal function $M_{w}$ defined by

$$
M_{w} f(x)=\sup _{Q \ni x} \frac{1}{w(Q)} \int_{Q}|f(y)| w(y) d y,
$$

where, as usual, the supremum is taken over all cubes, with sides parallel to the axes, containing $x$. By a Marcinkiewicz interpolation theorem in the context of the Lorentz spaces due to Calderón (see [BS, p. 225]), $M_{w}$ is bounded on $L^{r^{\prime}, 1}(w)$ with norm that we denote by $\left\|M_{w}\right\|_{L^{r^{\prime}, 1}(w)}$. Now we perform Rubio de Francia's algorithm in the following way:

$$
\mathcal{R}_{w} h(x)=\sum_{k=0}^{\infty} \frac{M_{w}^{k} h(x)}{2^{k}\left\|M_{w}\right\|_{L^{r^{\prime}, 1}(w)}^{k}} .
$$

Then, we have

(i) $h(x) \leq \mathcal{R}_{w} h(x)$.

(ii) $\left\|\mathcal{R}_{w} h\right\|_{L^{r^{\prime}, 1}(w)} \leq 2\|h\|_{L^{r^{\prime}, 1}(w)}=2$.

(iii) $M_{w}\left(\mathcal{R}_{w} h\right)(x) \leq 2\|M\|_{L^{r^{\prime, 1}(w)}} \mathcal{R}_{w} h(x)$, and thus $\mathcal{R}_{w} h \in A_{1}(w)$ (by this we mean that $\mathcal{R}_{w} h$ is a $A_{1}$-weight but with respect to the measure $\left.w(x) d x\right)$.

Thus, we know that $\mathcal{R}_{w} h \in A_{1}(w)$ and it is not difficult to show that $\mathcal{R}_{w} h w \in$ $A_{r} \subset A_{\infty}$ (see [CMP]). Besides, the estimate in $(c)$ holds in particular for $q$ and the $A_{\infty}$-weight $\mathcal{R}_{w} h w$. Thus,

$$
\begin{aligned}
\int_{\mathbb{R}^{n}} G f(x)^{q} h(x) w(x) d x & \leq \int_{\mathbb{R}^{n}} G f(x)^{q} \mathcal{R}_{w} h(x) w(x) d x \\
& \leq C \int_{\mathbb{R}^{n}} S f(x)^{q} \mathcal{R}_{w} h(x) w(x) d x \\
& \leq C\left\|(S f)^{q}\right\|_{L^{r, \infty}(w)}\left\|\mathcal{R}_{w} h\right\|_{L^{r^{\prime}, 1}(w)} \\
& \leq C\|S f\|_{L^{p, \infty}(w)}^{q}
\end{aligned}
$$

provided the second integral is finite, the fact that follows as in the previous sequence of inequalities. Then $(e)$ arises by taking the supremum over all functions $h$ as above.

The proof of $(d) \Longrightarrow(e)$ uses similar ideas that the reader can find in full detail in $\mathrm{CMP}$.

\section{Proof of Theorem 3.1}

In view of the previous extrapolation result if an estimate like (6) holds for some $0<p_{0}<\infty$, then the corresponding weak type-weak type (7) would hold for every $0<p<\infty$. On the other hand note that (8) applied to $w \in A_{1}$ - which means $M w(x) \leq w(x)$ a.e.- corresponds to $(c)$ in the latter result which implies (6). Besides, since $(d) \Longleftrightarrow(e)$, it suffices to disprove the weak type-weak type for some $0<p_{0}<\infty$. Let $1 \leq r<\infty$ and let $1 \leq t<r^{\prime}$. Choose $p_{0}$ such that $t<p_{0}<r^{\prime}$. Let us assume that (7) holds for $p_{0}$ and for all $w \in A_{1} \subset A_{\infty}$. Then, we have

$$
\|T f\|_{L^{p_{0}, \infty}(w)} \leq C\left\|M_{t} f\right\|_{L^{p_{0}, \infty}(w)} \leq C\|f\|_{L^{p_{0}}(w)}
$$


and $T$ is bounded from $L^{p_{0}}(w)$ to $L^{p_{0}, \infty}(w)$. In particular this estimate holds for the $A_{1}$ power weight $w(x)=|x|^{\alpha},-n<\alpha<-n p_{0} / r^{\prime}$, contradicting Theorem 3.2 .

\section{Proof of Theorem 3.2}

Let $\beta>0$ and consider the kernel $K(x)=k(|x|)$, where

$$
k(t)=t^{-\frac{n}{r}}\left(\log \frac{e}{t}\right)^{-\frac{1+\beta}{r}} \chi_{(0,1)}(t) .
$$

Note that $K \in L^{r}\left(\mathbb{R}^{n}\right)$. Take $0 \neq \eta \in \mathbb{R}^{n}$ far enough from the origin, for instance $|\eta|=4$. We define the kernel $\widetilde{K}(x)=K(x-\eta)$ and the operator $T$ as

$$
T f(x)=\widetilde{K} * f(x)=\int_{\mathbb{R}^{n}} K(x-\eta-y) f(y) d y .
$$

Observe that $\widetilde{K} \in L^{r}\left(\mathbb{R}^{n}\right) \cap L^{1}\left(\mathbb{R}^{n}\right)$ and hence the operator $T$ is bounded on $L^{q}\left(\mathbb{R}^{n}\right)$ for every $1 \leq q \leq \infty$. This kernel has the following property which will be proved later.

Lemma 6.1. The kernel $\widetilde{K}$ is in $H_{r}$.

Assume that $T$ maps $L^{p}\left(\mathbb{R}^{n},|x|^{\alpha}\right)$ into $L^{p, \infty}\left(\mathbb{R}^{n},|x|^{\alpha}\right)$ and we will get a contradiction. We write $w(x)=|x|^{\alpha}$. Let us take

$$
0<\varepsilon<-\alpha-\frac{n}{r^{\prime}} p \quad \text { and } \quad f(x)=|x+\eta|^{\frac{-n+\varepsilon}{p}} \chi_{B_{1}(-\eta)}(x) \in L^{p}\left(\mathbb{R}^{n}\right) .
$$

If $x \in B_{1}(-\eta)$, then $3<|x|<5$ and therefore

$$
\begin{aligned}
\sup _{\lambda>0} \lambda w\left\{x \in \mathbb{R}^{n}:|T f(x)|>\lambda\right\}^{\frac{1}{p}} & \leq C\left(\int_{\mathbb{R}^{n}}|f(x)|^{p}|x|^{\alpha} d x\right)^{\frac{1}{p}} \\
& \leq C 3^{\frac{\alpha}{p}}\left(\int_{\mathbb{R}^{n}}|f(x)|^{p} d x\right)^{\frac{1}{p}}<\infty .
\end{aligned}
$$

We define $h$ as

$$
h(t)=k(t) t^{\frac{-n+\varepsilon}{p}+n}=t^{\frac{\varepsilon}{p}+\frac{n}{r^{\prime}}-\frac{n}{p}}\left(\log \frac{e}{t}\right)^{-\frac{1+\beta}{r}} \chi_{(0,1)}(t) .
$$

Note that $\frac{\varepsilon}{p}+\frac{n}{r^{\prime}}-\frac{n}{p}<-\frac{\alpha}{p}-\frac{n}{p}<0$ and hence we can find $0<t_{0}<1$ such that $h(t)$ and $k(t)$ are strictly decreasing for $t \in\left(0, t_{0}\right)$. So, we write $\delta_{0}=\frac{2}{3} t_{0}$ and for $|x|<\delta_{0}$ we get

$$
\begin{aligned}
T f(x) & =\int_{|y|<1} K(x-y)|y|^{\frac{-n+\varepsilon}{p}} d y \geq \int_{|y|<|x| / 2} K(x-y)|y|^{\frac{-n+\varepsilon}{p}} d y \\
& \geq k\left(\frac{3|x|}{2}\right) \int_{|y|<|x| / 2}|y|^{\frac{-n+\varepsilon}{p}} d y
\end{aligned}
$$

where in the last estimate we have used that $|x-y|<\frac{3|x|}{2}<\frac{3 \delta_{0}}{2}=t_{0}$ and that $k$ is decreasing. Then, for $|x|<\delta_{0}$,

$$
T f(x) \geq C k\left(\frac{3|x|}{2}\right)|x|^{\frac{-n+\varepsilon}{p}+n}=C_{0} h\left(\frac{3|x|}{2}\right) .
$$


This estimate, and the fact that $h$ is strictly decreasing on the interval $\left(0, t_{0}\right)$, imply

$$
\begin{aligned}
\sup _{\lambda>0} \lambda^{p} w\left\{x \in \mathbb{R}^{n}:|T f(x)|>\lambda\right\} & \geq \sup _{\lambda>0} \lambda^{p} w\left\{|x|<\delta_{0}: C_{0} h\left(\frac{3|x|}{2}\right)>\lambda\right\} \\
& \geq C_{0}^{p} \sup _{\lambda>h\left(t_{0}\right)} \lambda^{p} w\left\{|x|<\delta_{0}: h\left(\frac{3|x|}{2}\right)>\lambda\right\} \\
& =C_{0}^{p} \sup _{0<t<t_{0}} h(t)^{p} w\left\{|x|<\frac{2 t}{3}\right\} \\
& =C \sup _{0<t<t_{0}} t^{\varepsilon+\alpha+\frac{n}{r^{\prime}} p}\left(\log \frac{e}{t}\right)^{-p \frac{1+\beta}{r}} \\
& =\infty,
\end{aligned}
$$

since $\varepsilon$ was taken such that $\varepsilon+\alpha+\frac{n}{r^{\prime}} p<0$. Observe that this contradicts (16).

Proof of Lemma 6.1. Since $\widetilde{K} \in L^{1}\left(\mathbb{R}^{n}\right) \subset H_{1}$ we may assume that $r>1$. Recall that $\widetilde{K}(x)=K(x-\eta)$, where $|\eta|=4$ and $K \in L^{r}\left(\mathbb{R}^{n}\right)$ is supported in the unit ball. In fact, these are the only hypotheses of this kernel that are going to come up in the following argument. Take $c=1$ and $R>|y|$. For $m \geq 1$ and $2^{m} R<|x| \leq 2^{m+1} R$, we have $2^{m-1} R<|x-y|<2^{m+2} R$. Then, it is easy to see that

$$
\begin{aligned}
A & =\sum_{m=1}^{\infty}\left(2^{m} R\right)^{\frac{n}{r^{\prime}}}\left(\int_{2^{m} R<|x| \leq 2^{m+1} R}|\widetilde{K}(x-y)-\widetilde{K}(x)|^{r} d x\right)^{\frac{1}{r}} \\
& \leq 2 \sum_{m=1}^{\infty}\left(2^{m} R\right)^{\frac{n}{r^{\prime}}}\left(\int_{2^{m-1} R<|x+\eta|<2^{m+2} R}|K(x)|^{r} d x\right)^{\frac{1}{r}} .
\end{aligned}
$$

Using the fact that the kernel is compactly supported, we observe that the number of non-zero terms in the previous summation is finite. Indeed, if $R \geq 5$, then for $m \geq 1$ and $x$ such that $2^{m-1} R<|x+\eta|<2^{m+2} R$, we have $|x| \geq 1$, and every integral vanishes. On the other hand, we assume that $R<5$ and let $m_{0}$ be the only non-negative integer such that $2^{m_{0}} R \leq 5<2^{m_{0}+1} R$. If $m \geq m_{0}+2$, whenever $2^{m-1} R<|x+\eta|<2^{m+2} R$ we get $|x|>1$ and therefore $K(x)=0$. Hence,

$$
\begin{aligned}
A & \leq 2 \sum_{m=1}^{m_{0}+1}\left(2^{m} R\right)^{\frac{n}{r^{\prime}}}\left(\int_{2^{m-1}} R<|x+\eta|<2^{m+2} R\right. \\
& \leq 2\|K\|_{L^{r}\left(\mathbb{R}^{n}\right)} \sum_{m=1}^{m_{0}+1}\left(2^{m} R\right)^{\frac{n}{r^{\prime}}} \approx\|K\|_{L^{r}\left(\mathbb{R}^{n}\right)}\left(2^{m_{0}} R\right)^{\frac{n}{r^{\prime}}} \approx\|K\|_{L^{r}\left(\mathbb{R}^{n}\right)} .
\end{aligned}
$$

\section{ACKNOWLEDGMENT}

The authors thank Professor J. Duoandikoetxea for the interest showed in the paper and for pointing out that the original proof could be simplified by means of Theorem 3.2 .

\section{REFERENCES}

[AP] J. Álvarez and C. Pérez, Estimates with $A_{\infty}$ weights for various singular integral operators, Boll. Un. Mat. Ital. A (7) 8 (1994), no. 1, 123-133. MR95f:42027

[BS] C. Bennett and R. Sharpley, Interpolation of Operators, Academic Press, New York, 1988. MR.89e:46001 
[Chr] M. Christ, Lectures on Singular Integral Operators, Reg. Conferences Series in Math. 77, Amer. Math. Soc., Providence, 1990. MR 92f:42021

[Coi] R. Coifman, Distribution function inequalities for singular integrals, Proc. Acad. Sci. U.S.A. 69 (1972), 2838-2839. MR 46:2364

[CMP] D. Cruz-Uribe, J.M. Martell, C. Pérez, Extrapolation results for $A_{\infty}$ weights and applications, to appear in J. Funct. Anal.

[Duo] J. Duoandikoetxea, Fourier Analysis, American Math. Soc., Grad. Stud. Math. 29, Providence, RI, 2000. MR2001k:42001

[GR] J. García-Cuerva and J.L. Rubio de Francia, Weighted Norm Inequalities and Related Topics, North-Holland Math. Stud. 116, North-Holland, 1985. MR87d:42023

[Gra] L. Grafakos, Estimates for maximal singular integrals with rough kernels, Colloq. Math. (to appear).

[Hof] S. Hofmann, Singular integrals with power weights, Proc. Amer. Math. Soc. 110 (1990), no. 2, 343-353. MR90m:42026

[J] F. John, Quasi-isometric mappings, Seminari 1962/63 Anal. Alg. Geom. e Topol., vol. 2, Ist. Naz. Alta Mat., (1965), 462-473. MR32:8315

$[\mathrm{KW}]$ D.S. Kurtz and R.L. Wheeden, Results on weighted norm inequalities for multipliers, Trans. Amer. Math. Soc. 255 (1979), 343-362. MR81j:42021

[Ler] A.K. Lerner, Weighted norm inequalities for the local sharp maximal function, preprint (2002).

[MW] B. Muckenhoupt and R. L. Wheeden, Weighted norm inequalities for singular and fractional integrals, Trans. Amer. Math. Soc. 161 (1971), 249-258. MR44:3155

[Pe1] C. Pérez, Weighted norm inequalities for singular integral operators, J. London Math. Soc. 49 (1994), 296-308. MF.94m:42037

[Pe2] C. Pérez, On sufficient conditions for the boundedness of the Hardy-Littlewood maximal operator between weighted $L^{p}$-spaces with different weights, Proc. of the London Math. Soc. (3) 71 (1995), 135-157. MR 96k:42023

[PT] C. Pérez and R. Trujillo-González, Sharp weighted estimates for multilinear commutators, Journal of the London Mathematical Society 65 (2002), 672-692. MR.2003f:42022

[RRT] J.L. Rubio de Francia, F.J. Ruiz and J.L. Torrea, Calderón-Zygmund theory for operatorvalued kernels, Adv. Math. 62 (1986), 7-48. MR88f:42035

[S] J.-O. Strömberg, Bounded mean oscillation with Orlicz norms and duality of Hardy spaces, Indiana Univ. Math. J. 28 (1979), 511-544. MR81f:42021

[Ste] E.M. Stein, Harmonic Analysis: Real-Variable Methods, Orthogonality and Oscillatory Integrals, Princeton University Press, New Jersey, USA, 1993. MR.95c:42002

[Wat] D.K. Watson, Weighted estimates for singular integrals via Fourier transform estimates, Duke Math. J. 60 (1990), no. 2, 389-399. MR91b:42035

[Wil] J.M. Wilson, Weighted norm inequalities for the continuous square functions, Trans. Amer. Math. Soc. 314 (1989), 661-692. MR91e:42025

Departamento de Matemáticas, C-XV, Universidad Autónoma de Madrid, 28049 MADRID, SPAIN

E-mail address: chema.martell@uam.es

Departamento de Análisis Matemático, Facultad de Matemáticas, Universidad de Sevilla, 41080 Sevilla, Spain

E-mail address: carlosperez@us.es

Departamento de Análisis Matemático, Universidad de La Laguna, 38271 La Laguna - S/C de Tenerife, Spain

E-mail address: rotrujil@ull.es 